\title{
Electromyogram-based neural network control of transhumeral prostheses
}

\author{
Christopher L. Pulliam, MS; ${ }^{1 *}$ Joris M. Lambrecht, MS; ${ }^{1}$ Robert F. Kirsch, PhD ${ }^{1-2}$ \\ ${ }^{1}$ Department of Biomedical Engineering, Case Western Reserve University, Cleveland, $\mathrm{OH} ;{ }^{2}$ Center of Excellence in \\ Functional Electrical Stimulation, Louis Stokes Cleveland Department of Veterans Affairs Medical Center, Cleveland, OH
}

\begin{abstract}
Upper-limb amputation can cause a great deal of functional impairment for patients, particularly for those with amputation at or above the elbow. Our long-term objective is to improve functional outcomes for patients with amputation by integrating a fully implanted electromyographic (EMG) recording system with a wireless telemetry system that communicates with the patient's prosthesis. We believe that this should generate a scheme that will allow patients to robustly control multiple degrees of freedom simultaneously. The goal of this study is to evaluate the feasibility of predicting dynamic arm movements (both flexion/extension and pronation/supination) based on EMG signals from a set of muscles that would likely be intact in patients with transhumeral amputation. We recorded movement kinematics and EMG signals from seven muscles during a variety of movements with different complexities. Time-delayed artificial neural networks were then trained offline to predict the measured arm trajectories based on features extracted from the measured EMG signals. We evaluated the relative effectiveness of various muscle subsets. Predicted movement trajectories had average root-mean-square errors of approximately $15.7^{\circ}$ and $24.9^{\circ}$ and average $R^{2}$ values of approximately 0.81 and 0.46 for elbow flexion/extension and forearm pronation/supination, respectively.
\end{abstract}

Key words: amputation, artificial neural network, control, electromyographic, myoelectric, myoelectric control, pattern recognition, prosthesis, prosthetic limb, transhumeral.

\section{INTRODUCTION}

Upper-limb amputation can cause a great deal of functional impairment for the estimated 41,000 individuals in the United States living with major upper-limb loss (i.e., excluding loss of only fingers) [1]. While a person may choose to wear only a passive or cosmetic prosthesis in place of the amputated arm segments, active prostheses are capable of restoring function beyond acting as an opposition device. Body-powered prostheses are powered and controlled by gross body movements. These movements, usually of the shoulder, upper arm, or chest, are captured by a harness system attached to a cable that is connected to a terminal device (or, depending on the level of amputation, to a prosthetic wrist and/or elbow system). Electrically powered prostheses use small electrical motors rather than motion of other body parts to power movements of the terminal device (hand or hook), wrist, and elbow. Such systems are frequently controlled by recording electrical signals-known as myoelectric signals (MES) or electromyographic (EMG) signalsgenerated by the contractions of residual muscles using electrodes placed on the surface of the skin over the muscles, but they can also be controlled by body movements

\footnotetext{
Abbreviations: ADL = activity of daily living, $\mathrm{ANN}=$ artificial neural network, ANOVA = analysis of variance, $\mathrm{DOF}=$ degree of freedom, EMG = electromyographic, FE = flexion/ extension, LED = light-emitting diode, MES = myoelectric signal, PS = pronation/supination, RMSE = root-mean-square error, TDANN = time-delayed artificial neural network.

*Address all correspondence to Christopher L. Pulliam, MS; Case Western Reserve University, Wickenden Building 311, 2071 Martin Luther King Jr. Dr, Cleveland, OH 44106; 216-368-8541; fax: 216-368-4872.

Email: christopher.pulliam@case.edu

DOI:10.1682/JRRD.2010.12.0237
} 
by using linear potentiometers, buttons, force-sensitive resistors, or switches. The goal of this work is to evaluate the feasibility of achieving EMG-based, simultaneous, multiple-joint control of transhumeral prostheses. This is part of a larger effort to integrate fully implanted EMG recording with a wireless telemetry system that communicates with the patient's prosthesis.

EMG signals have proven to be effective command sources for the control of externally powered upper-limb prostheses. Most commercially available prosthetic systems use a relatively simple control scheme, whereby the amplitude of EMG signals recorded from two sites beneath the socket are used to actuate one of the motors embedded in the prosthesis. This allows only a single degree of freedom (DOF) to be operated at a time and requires a mode switch to transition between operating the various DOFs of the system. Normal human arm functions use coordinated, simultaneous movements of the hand, wrist, and elbow to interact with the environment. These coordinated, simultaneous patterns of movement are particularly prevalent in many activities of daily living (ADLs) such as feeding and grooming. When contrasted with normative arm function, the sequential control method seen in prosthetic arms can be frustratingly slow and unnatural. While there has been a great deal of recent development in the mechanical design of prosthetic arms [1], a highly articulated limb is of little use if its movements are not well coordinated or if it is difficult to operate.

Approximately 20 percent of all individuals with upper-limb amputation never seek out a prosthesis, and as many as half of those who do receive a prosthesis will ultimately abandon the technology [2]. A number of reasons exist for this poor compliance rate; several are related to the perception that the functional gain is limited relative to the awkwardness of the available control interfaces and the accompanying poor performance of the prosthesis. To improve functional outcomes for individuals with high-level upper-limb amputation, researchers must develop a more sophisticated approach to MES control. There are two general goals for these refined methods: (1) more information must be extracted from the EMG signals, and (2) a controller should simultaneously identify all intended motions of the patient [3].

Feature extraction and other advanced signal processing techniques have been applied extensively to MES control problems to increase the information yield from EMG signals. Schemes to identify intended movements have been developed that rely on features such as simple time-domain statistics [4], autoregressive coefficients [5], and wavelet coefficients [6] to extract information from EMG signals relevant to the motion intent of a patient. Another approach for obtaining more information for inferring movement intent is to increase the number of muscle EMG signals that are recorded. Commercially available upper-limb prostheses typically include just two EMG recording channels, which are usually applied to antagonist muscles (e.g., biceps and triceps) to control one prosthesis DOF at a time. Research systems have evaluated the use of significantly more EMG channels. As early as the 1970s, researchers demonstrated that the use of multicontact electrode recordings (i.e., 8 to 10 EMG channels) with appropriate control schemes was able to improve a system's ability to distinguish as many as 10 distinct types of movement, with accuracies ranging from 70 to 98 percent [7].

Current MES control systems typically employ bipolar surface recordings because the electrodes are noninvasive, inexpensive, and readily incorporated into the socket of the prosthesis. However, surface electrodes also have a number of drawbacks, including a limited ability to selectively record from different muscles, changes in signal magnitude and frequency content caused by changes in the skin-electrode interface associated with environmental and physiological conditions (e.g., skin moisture), and difficulty achieving consistent day-to-day electrode placement. Intramuscular EMG recordings would potentially address these limitations and be particularly attractive if paired with an implanted recording and telemetry system that eliminated all cabling and physical connections. Such a system would also have the benefit of accessing not only muscles directly beneath the socket but also deeper muscles and muscles that are distant from the site of amputation. Implanted EMG recordings have been used successfully for functional electrical stimulation applications [8-9] and are being pursued for upper-limb prosthesis applications [10]. While no increases in the accuracy of transradial pattern-recognition-based classifiers have been demonstrated when using intramuscular EMG recordings in acute laboratory experiments [11-12], it is important to note that these findings may not hold for other levels of amputation. Even for transradial prostheses, many of the clinical advantages of intramuscular recordings described previously would only be apparent in long-term evaluations, which have been outside the scope of previous studies. 
A number of different approaches have been taken to design a "controller" that maps features from the EMG signals to the desired movements of the prosthesis. Discrete limb-movement types or "states" (e.g., elbow flexion vs extension) are often identified using pattern-recognition approaches such as linear discriminant analysis [13], fuzzy logic [14-15], and artificial neural networks (ANNs) $[4,16]$. Alternatively, ANNs have been used to predict continuous movement trajectories using EMG signals rather than discrete states. The continuous prediction of trajectories has the potential advantage of allowing coordinated and simultaneous control of multiple DOFs in a natural manner. Cheron et al. demonstrated that a dynamic, recurrent ANN using EMG recordings from several relevant shoulder and elbow muscles directly as inputs (rather than extracted features of the EMG) could be trained to predict arm endpoint trajectories [17]. Sebelius et al. demonstrated ANN prediction of hand and wrist movement trajectories in the control of a virtual hand [18]. Au and Kirsch developed a time-delayed ANN (TDANN) that used EMG signals from several shoulder and arm muscles to predict three shoulder motions and elbow flexion/extension (FE) [19]. While the aim of Au and Kirsch was to investigate an approach to developing advanced control systems for restoring shoulder and elbow movements to individuals with tetraplegia using functional electrical stimulation of paralyzed muscles, the results are also directly relevant for above-elbow prostheses. Au and Kirsch predicted the four joint angles with average root-mean-square errors (RMSEs) of $<20^{\circ}$, establishing that muscles in the upper arm and shoulder contain significant information related to elbow and shoulder kinematics. Control of wrist rotation (i.e., forearm pronation/supination [PS]) as well as elbow FE must demonstrate complete user interface for transhumeral prostheses, which will likely require a different set of muscle EMG recordings.

The work presented here specifically investigates the use of TDANNs to predict dynamic arm movements (both FE and PS) based on EMG features extracted from a set of muscles that would be available to an implanted system for patients with transhumeral amputation. We evaluated the efficacy of using different numbers of muscle EMG signals and the relative advantages of recording from specific muscles in predicting the continuous movement of two arm motions. Finally, we explored the design of an optimal TDANN that would be used to implement an advanced MES controller.

\section{METHODS}

\section{Subject Information}

Five nondisabled male subjects between the ages of 24 and 30 took part in this study. All subjects gave informed consent to the procedures as approved by the MetroHealth Medical Center Institutional Review Board.

\section{General Approach and Experimental Setup}

Figure 1 summarizes the overall approach used in this study. We conducted experiments in which we simultaneously recorded upper-limb kinematics and EMG signals during a series of arm movements performed by nondisabled subjects (Figure 1(a)). TDANNs were then trained offline to predict measured arm trajectories based on features extracted from the measured EMG signals (Figure 1(b)).

\section{Motion Data Acquisition}

An Optotrak Certus motion capture system (Northern Digital Inc; Waterloo, Canada) with two position sensor units recorded the three-dimensional motions of the arm (Figure 1(a)) as has been previously described [20]. We fixed light-emitting diode (LED) marker clusters over the upper arm and forearm to track the motion of the body segments. During each trial, the subject was positioned in such a way that arm movements throughout the workspace were unobstructed and at least one of the sensor units could view each marker cluster. We established the positions of a set of bony landmarks relative to the positions of the marker clusters by palpating and digitizing the locations of these points using the LED-instrumented stylus provided with the Optotrak Certus motion capture system. The Optotrak Certus motion capture system software then automatically calculated the global positions of these bony landmarks for each frame. Subjects performed several movements as data were collected, including (1) single-joint movements, (2) multiple-joint movements, and (3) a set of simulated ADLs. During each single-joint movement trial, either FE or PS were performed cyclically with the shoulder in one of three distinct postures: at the subject's side, abducted $90^{\circ}$, or flexed $90^{\circ}$. Additional pronosupination single-joint movement trials were collected with the arm at the subject's side and the elbow flexed $90^{\circ}$. Subjects subsequently repeated all single-joint movement trials while holding a mass of $1 \mathrm{~kg}$ in the hand. Multiple-joint movement trials included serial movements (e.g., shoulder abduction, then elbow flexion, 


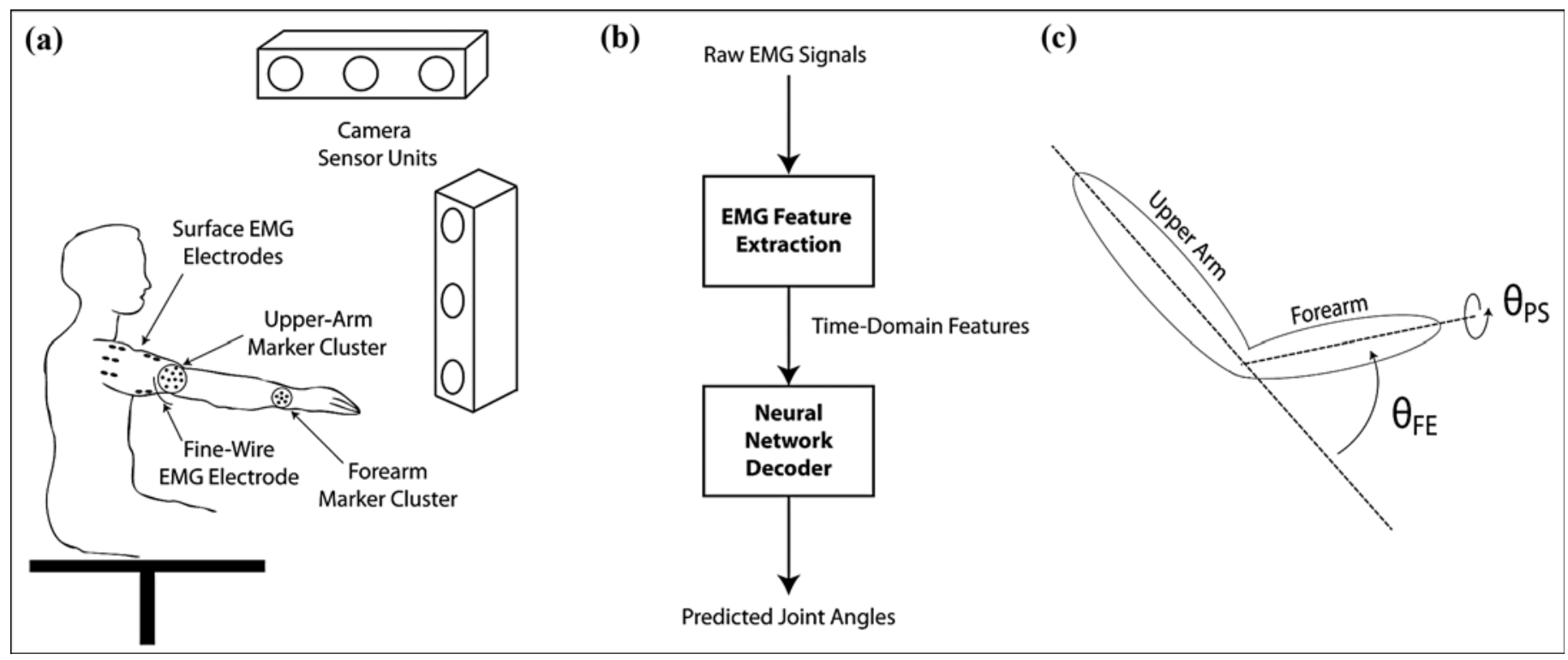

Figure 1.

(a) Movement kinematics and electromyographic (EMG) signals recorded during variety of movements from several muscles in nondisabled upper arm and shoulder. (b) Block diagram of study approach. Time-delayed artificial neural networks were trained to predict measured movement trajectories based on features extracted from measured EMG signals. (c) Definitions of joint angles in this study. $\theta_{\mathrm{FE}}=$ flexion and extension of elbow, $\theta_{\mathrm{PS}}=$ pronation and supination of forearm.

then supination, then pronation, then elbow extension, then shoulder adduction), simultaneous movements (e.g., simultaneous elbow flexion and supination followed by simultaneous elbow extension and pronation), and reaches toward imaginary points in space that spanned the workspace of the arm (returning the arm to the initial position on an armrest between consecutive reaches). The simulated ADLs included feeding, hair brushing, and opening a door. All kinematics were recorded at $30 \mathrm{~Hz}$.

\section{Electromyographic Signal Acquisition}

EMG signals were recorded from seven muscles for each subject: brachialis, biceps, medial head of triceps, posterior deltoid, anterior deltoid, middle deltoid, and clavicular pectoralis major. A physician inserted bipolar percutaneous ("fine-wire") intramuscular electrodes $(0.05 \mathrm{~mm}$ diameter insulated nickel alloy wires with exposed tips for recording) into the belly of the brachialis. The brachialis EMG was recorded intramuscularly because it is inaccessible from the surface. Intramuscular electrode placement was verified by observing EMG activity during manual muscle tests in which the subject attempted to (1) flex the elbow against resistance with the forearm fully pronated, then with the forearm fully supinated, and (2) supinate the forearm against resistance with the elbow flexed slightly. The electrode was concluded to be in the correct location if there was (1) no significant change in the EMG amplitude between flexing the elbow with the forearm pronated compared with flexing the elbow with the forearm supinated and (2) no increase in activity as the subject supinated the forearm. Failure of either of these tests would be consistent with the electrode being in deep portions of the biceps rather than the brachialis. When this occurred, the physician advanced the cannula deeper into the arm and the tests were repeated to verify placement in the brachialis. Two disposable $2.2 \mathrm{~cm}^{2}$ surface electrodes placed approximately $2.5 \mathrm{~cm}$ apart (center-to-center) over the belly of each muscle obtained differential surface EMG recordings from the remaining six muscles. The exact locations were determined by palpation and visual inspection, consistent with the methods outlined in an EMG reference [21]. All EMG signals were amplified, alternating-current coupled, and low-pass filtered with a $1 \mathrm{kHz}$ cutoff using CED amplifiers (model 1902, Cambridge Electronic Design Limited; Cambridge, United Kingdom) and then sampled at $2.5 \mathrm{kHz}$ with a data acquisition card (PCIM-DAS1602, Measurement Computing Corporation; Norton, Massachusetts) installed in a computer running xPCTarget (MathWorks; Natick, Massachusetts). 
Proprietary Optotrak software (Northern Digital Inc) recorded the positions of the bony landmarks of the arm during these trials. A custom MATLAB/xPCTarget interface (MathWorks) was used for visualization of EMG signals, synchronization of EMG and motion acquisition with a hardware trigger, and data storage. Approximately 50 to 60 movement trials were recorded during each session. In total, each experiment lasted about 4 hours, including rest time between trials.

\section{Data Processing}

The digitized EMG data sets were then processed offline by filtering, windowing, and extracting signal features. The raw signals were high-pass filtered with a fifth-order Butterworth digital filter with a cutoff frequency of $10 \mathrm{~Hz}$ to remove movement artifacts. Several features were then extracted from 320-sample (128 ms) rectangular windows of these signals with 50 percent overlap between adjacent segments, producing an effective sample time of $64 \mathrm{~ms}$. The time-domain statistics described by Hudgins et al. were used [4]. Specifically, the mean absolute value, waveform length, number of zero crossings, and number of slope sign changes were extracted from each window, generating a four-element feature set for each EMG channel.

The locations of the LED markers measured by the Optotrak Certus motion capture system were processed (relative to known bony landmark locations) to obtain the orientations of the humerus and forearm, and then the various joint angles of the arm from each movement trial. To do this, we used the locations of the bony landmarks used to generate local coordinate systems attached to each bone segment. The relative orientations of these local bone coordinate systems were used to compute the elbow FE and forearm PS joint angles. $\theta_{\mathrm{FE}}$ is defined as $0^{\circ}$ when the elbow is fully extended, while $\theta_{\mathrm{PS}}$ is defined as $0^{\circ}$ when the forearm is fully supinated (Figure 1(c)). The joint angle trajectories were then digitally upsampled with linear interpolation from $30 \mathrm{~Hz}$ to $2.5 \mathrm{kHz}$ (to match the sampling frequency of the raw EMG). After upsampling, the joint angles were then windowed exactly as the raw EMG signals (i.e., 320-sample rectangular windows with $50 \%$ overlap between adjacent segments). The average joint angle values were extracted from each window, effectively downsampling the motion data such that its sample time matched that of the EMG features.

\section{Neural Network Training and Testing}

We investigated using a TDANN to predict FE and PS joint-angle trajectories based on EMG information obtained from muscles that should be intact and available in patients with transhumeral amputation. We used a twolayer feed-forward structure with a nonlinear tangentsigmoidal activation function for the hidden layer and a linear output layer. This structure is known to be capable of characterizing any static nonlinear relationship [22], such as that between EMG and muscle force or joint position. The use of delayed input signals enables the neural network to capture dynamic input-output properties and account for the delay between onset of EMG activity and mechanical arm movement. TDANNs have the additional advantage of rapid training time when compared with dynamic neural networks with recurrent connections. All TDANNs were trained using MATLAB's Neural Network Toolbox (MathWorks).

We used several strategies during TDANN training to achieve good generalization, despite expected variations in the muscle activation patterns over time caused by small changes in movement strategy and fatigue. We used a repeated, random subsampling method for cross-validation. This method randomly splits the data set into training and testing data-here, we randomly selected 10 to 12 trials as the independent test data set and used the remaining trials for training. The process was constrained such that each test set was guaranteed to have representative trials (randomly selected for each split) from each of the four categories of movement-single-joint movements with no load, singlejoint movements with load, simultaneous and sequential movements, and simulated ADLs. The remaining trials (which comprised the training data set) were then used to train the neural network. All trials in the training set were split such that only the first 80 percent of each trial was presented to the backpropagation algorithm. We used the remaining data (i.e., the last $20 \%$ of each trial in the training set) during training to monitor the TDANN's ability to generalize to novel inputs and prevent memorization. Thus, we stopped the training when the error for this subset of training data did not improve or increase for 50 consecutive training iterations. All networks were trained in batch mode. The weights of the network with the minimum training error were saved as the final network structure. We subsequently used the testing data set to evaluate the predictive ability of the trained network on novel inputs. This process was repeated five times (i.e., a 5-split cross-validation). Each network was trained two different times to account for 
different random initialization weights. We used the network with better performance in subsequent analyses. All results reported in this article are the cross-validated averages over the five splits of the network.

The number of neurons used in the hidden layer was varied systematically between 5 and 80 while we monitored the prediction error. We also investigated using a variable time history of the EMG features as inputs to the TDANN. To determine the extent of the time history of the features required to most accurately predict the joint angles, we varied the number of delays from 1 to 15 (64$960 \mathrm{~ms}$ ). The goal was to find the smallest TDANN capable of providing good prediction results, which would prevent memorization of the inputs and allow efficient, realtime implementation in a prosthetic limb in the future. Selecting a "simpler" TDANN also avoids the degradation in performance one would expect in larger networks because of their increased dimensionality and the limited amount of training data. The maximum number of TDANN training iterations and the minimum error goal were chosen heuristically based on the performance observed during training. The performance of all the trained networks was quantified as the ability to predict the joint-angle data from the testing data set. The goodness of fit of the TDANN was summarized by the RMSE and the coefficient of determination $\left(R^{2}\right)$ between the experimentally recorded joint-angle trajectories and the corresponding trajectories predicted by the TDANN. RMSE is in physical units (in this case, degrees) and thus has more direct functional implications. $R^{2}$ is a normalized goodness of fit measure that indicates the correspondence between actual and predicted joint trajectories regardless of their magnitude.

We used a two-way repeated-measures analysis of variance (ANOVA) with the number of hidden layer neurons and the number of input delays as factors to determine whether changing the structure produced significant changes in network performance. Separate analyses were run with each of the performance metrics (described previously) as the response variables. Multiple comparisons with Bonferroni corrections $(\alpha=0.05)$ were employed post hoc to identify which architecture was just large enough for accurate joint-angle prediction. This architecture was used for all remaining analyses. After identifying an appropriate architecture, we used a one-way repeatedmeasures ANOVA with multiple comparisons and Bonferroni corrections to determine whether the performance was consistent across all categories of movements included in the study.

\section{Muscle Subset Selection}

While the EMG signals were recorded from a total of seven muscles in the upper arm and shoulder region during experimental sessions, it is possible that not all of them are actually useful for predicting FE and PS joint angles. Keeping in mind that increasing the number of muscles has an associated cost in terms of surgical complexity and device expense, we examined reduced subsets of muscles to determine whether they would be sufficient for movement trajectory prediction. We evaluated the relative effectiveness of various muscle subsets by implementing a forward-selection algorithm. At each step of this algorithm, each muscle that was not already a part of the set was tested for inclusion by adding the EMG features from that muscle to the neural network input and repeating the training process. The muscle that had the largest positive effect was then retained as a part of the input set and the algorithm repeated the process with the remaining muscles in the next step. Thus, we began with a subset that included the muscle that had the most significant contribution to network prediction and continued adding muscles until all seven were included, resulting in an ordinal set of muscles for each subject. We used a one-way repeated-measures ANOVA and multiple comparisons with Bonferroni corrections ( $\alpha=0.05)$ to determine the minimum number of input muscles that provided performance that did not statistically differ from the maximal observed performance. Effectively, this was the number of muscles such that adding more muscles did not statistically improve performance, as quantified by a statistically significant increase in the $R^{2}$ or decrease in the RMSE.

Since it would be advantageous to use a fixed set of muscles in a clinical system rather than tailoring the surgical targets to each patient, we also considered a single ordered muscle set based on the average ranks across all of the subjects. We used a two-way repeated-measures ANOVA (the number of muscles and subset type-fixed vs subject-specific - as factors) with multiple comparisons and appropriate Bonferroni corrections to determine whether fixing the muscle set has a significant effect on predictive accuracy. 


\section{RESULTS}

Figure 2 illustrates the EMG (top seven panels) and kinematic (lower two panels) data recorded during a typical experimental trial. During this trial, the subject reached for a series of imaginary targets in space that spanned the workspace of the arm, returning the arm to a resting position on the leg between reaches. Features were extracted from windows of EMG recordings of the seven muscles and the TDANN used them to predict the joint angles across the many trials of a given experiment.

\section{Optimization of Neural Network Architecture}

We varied a number of architectural parameters of the TDANN to determine an optimal structure for predicting the FE and PS angles based on the features calculated from the windows of EMG data. Figure 3 shows the effects of varying the number of hidden layer neurons and the number of input time delays on average jointangle prediction performance for all subjects. Generally, increasing the number of hidden layer neurons and the number of input time delays resulted in an increase in the accuracy of prediction as quantified by statistically significant changes in $\mathrm{RMSE}_{\mathrm{FE}}, \mathrm{RMSE}_{\mathrm{PS}}, R_{\mathrm{FE}}^{2}$, and $R_{\mathrm{PS}}^{2}$ $(p<0.001)$ for both factors. For all metrics, the interaction between the number of hidden layer neurons and the number of input time delays was not statistically significant $(p>0.50)$, so we considered them independently when selecting the optimal value of each parameter.

We used multiple comparisons with Bonferroni corrections to determine the specific architecture to be used. There were no significant differences in $\mathrm{RMSE}_{\mathrm{FE}}(p>$ $0.28)$, $\operatorname{RMSE}_{\mathrm{PS}}(p>0.21)$, or $R_{\mathrm{FE}}^{2}(p>0.12)$ when the number of hidden layer neurons was increased beyond 10. $R_{\text {PS }}^{2}$, however, showed a statistically significant increase when the number of neurons was increased from 10 to 20, but did not show any significant changes $(p>$ 0.99 ) when increased beyond 20 . With respect to the number of input time delays, both $\mathrm{RMSE}_{\mathrm{FE}}$ and $R_{\mathrm{FE}}^{2}$ increased progressively as the number of past values increased from 1 to 5 , but we saw no further improvement $(p>0.10)$ beyond 5 past values (i.e., $320 \mathrm{~ms}$ of time history). No statistically significant increases $(p>0.11)$ in either RMSE $E_{\mathrm{PS}}$ or $R_{\mathrm{PS}}^{2}$ were found when increasing the number of input delays beyond 7 (i.e., $448 \mathrm{~ms}$ of time history). As there was no significant benefit to including more than 20 hidden layer neurons or 7 input time delays for any of the performance metrics, we selected these to be the parameter values for subsequent analyses.
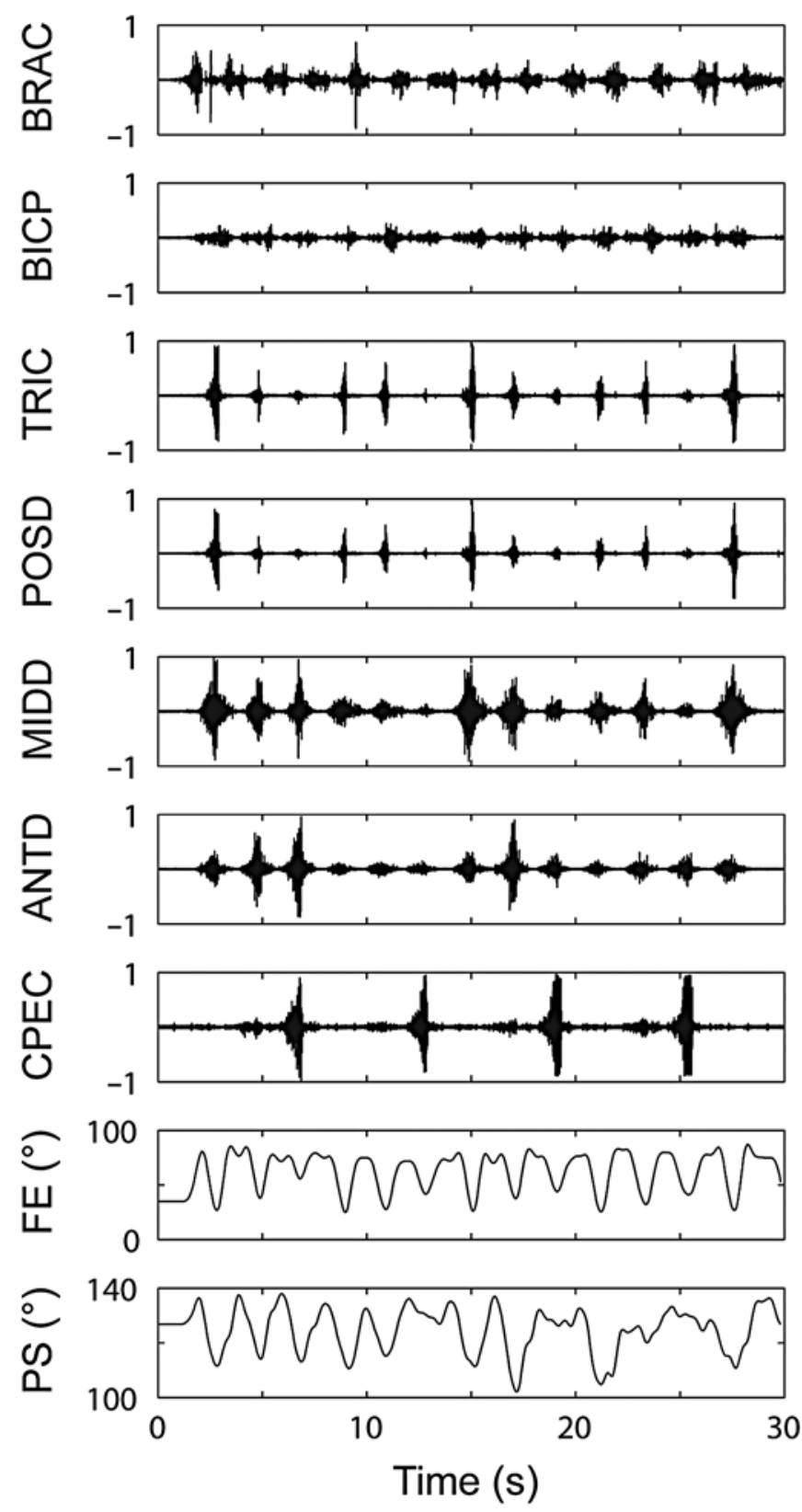

Figure 2.

Experimental data recorded during single trial during which subject reached for series of imaginary targets in space, returning arm to rest position between reaches. ANTD = anterior deltoid, BICP = biceps, BRAC = brachialis, $\mathrm{CPEC}=$ clavicular pectoralis, $\mathrm{FE}=$ flexion $/$ extension, MIDD = middle deltoid, POSD = posterior deltoid, PS = pronation/supination, TRIC $=$ triceps. 

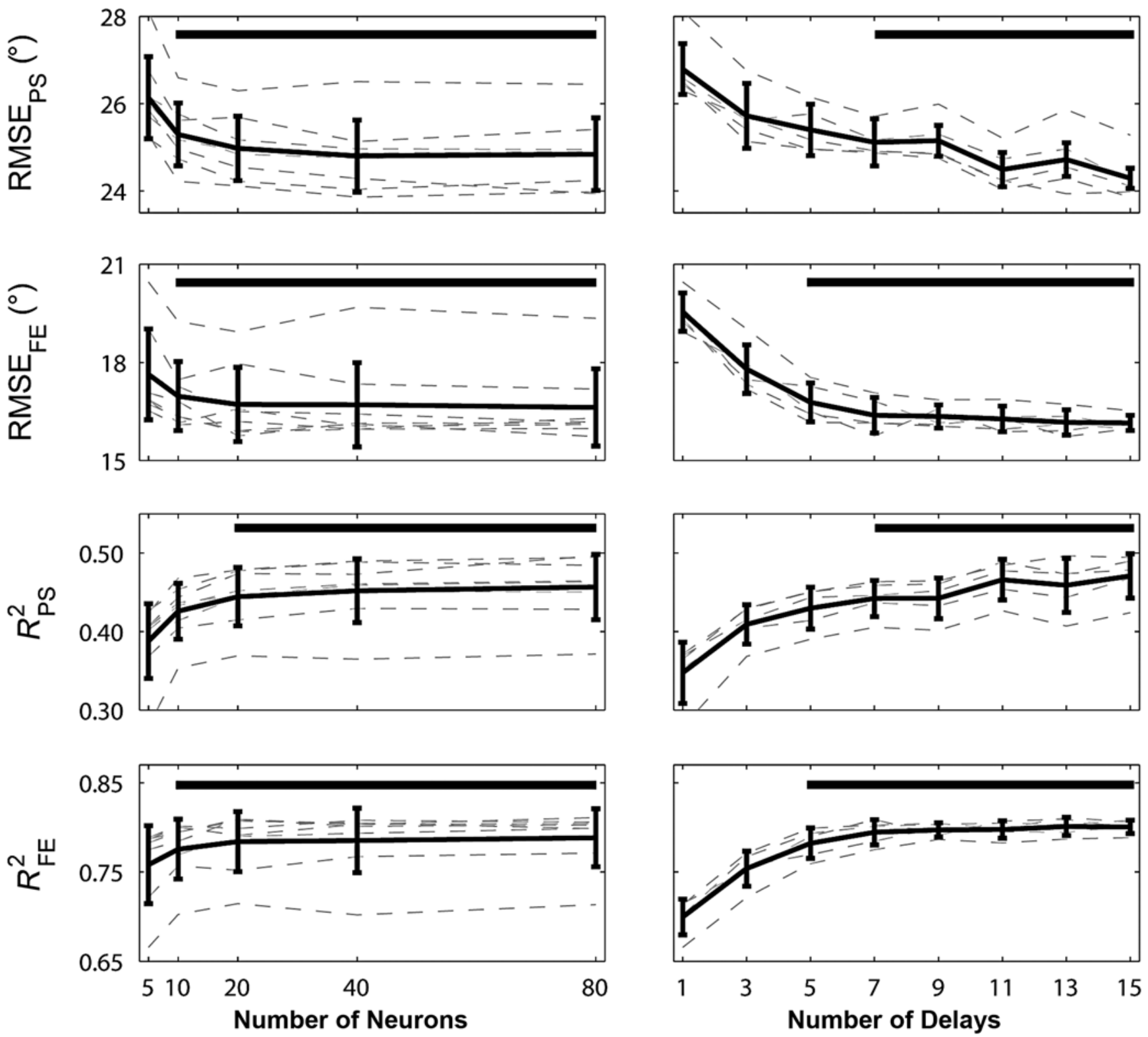

Figure 3.

Optimization of neural network architecture. Cross-validated performance is shown as function of number of hidden layer neurons (left) and input time delays (right). Light dotted lines correspond to networks trained with specific numbers of neurons (left) or input delays (right). Solid bold lines indicate mean \pm standard deviation. Solid black vertical bars indicate range of values that provided optimal performance. FE $=$ flexion/ extension, $\mathrm{PS}=$ pronation/supination, $\mathrm{RMSE}$ = root-mean-square error.

\section{Time-Delayed Artificial Neural Network Prediction of Movement Trajectories}

Once an adequate neural network structure was identified, we used the features extracted from the EMG recordings as inputs to assess their overall ability to predict the joint-angle trajectories. Figure 4 illustrates the ability of a
TDANN with 20 hidden layer neurons and 7 input delays to simultaneously predict the FE and PS joint angles from EMG recordings from a single subject for a variety of different arm movements. The upper row of panels illustrates TDANN joint-angle predictions (black line) for elbow FE, while the lower row of panels illustrates forearm PS 


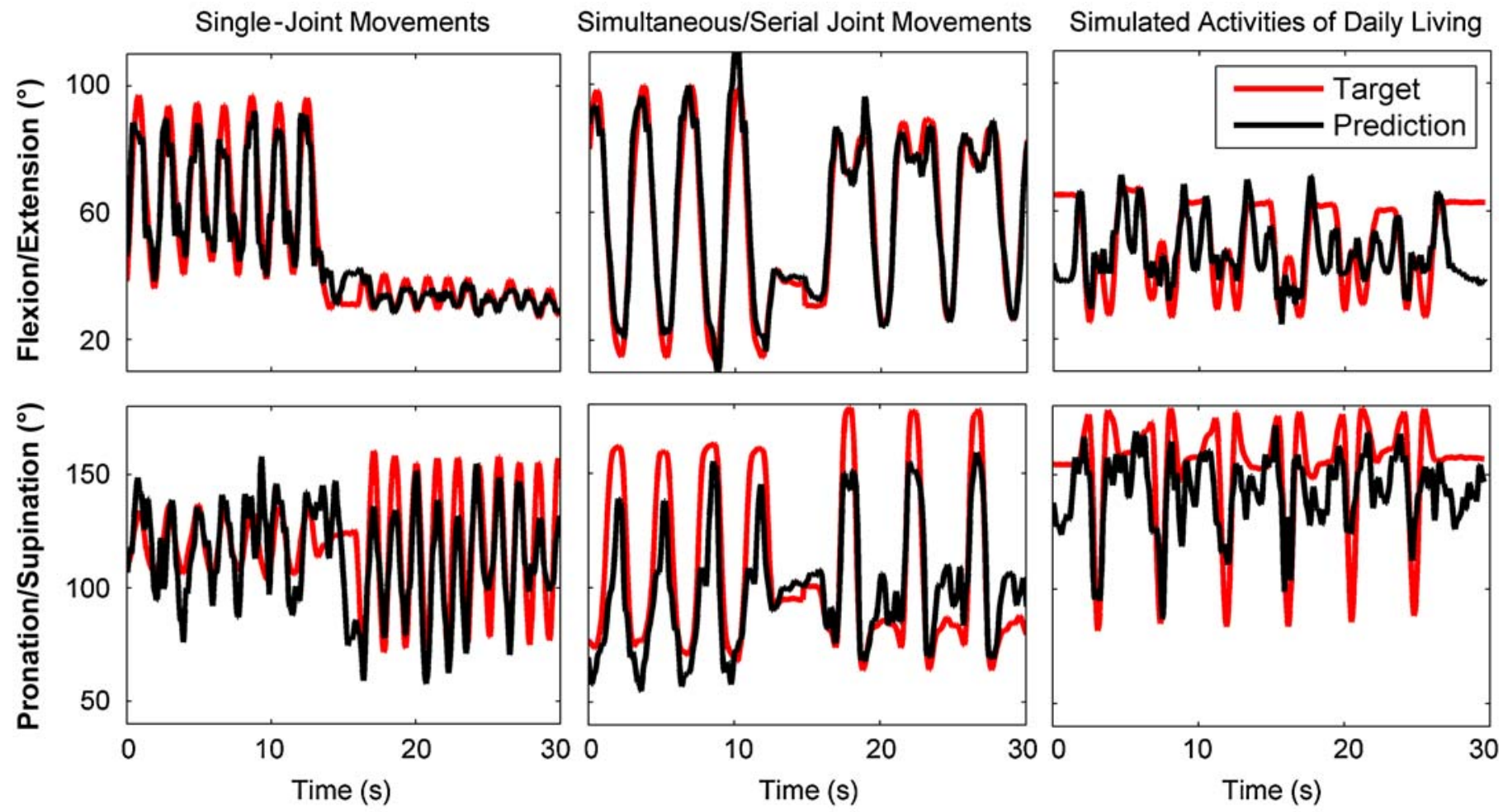

Figure 4.

Prediction performance of time-delayed artificial neural network (TDANN) for flexion/extension and pronation/supination joint angles for variety of movements (subject 5). Each column represents different movement type, with each row representing different degree of freedom. Several different movements were performed during each $30 \mathrm{~s}$ trial. Within each panel, experimentally recorded trajectory (red line) is overlaid with predictions from TDANN (black line).

performance. The left column of panels illustrates movements during which one joint was moved at a time, the center column illustrates a combination of serial and simultaneous movements, and the right column illustrates simulated ADLs-all predicted by the same TDANN. Note that the data plotted in each panel represents several movements.

Table 1 summarizes the cross-validated average RMSE and $R^{2}$ values for each joint angle and each subject for different movement types. The bottom two rows of the table summarize performance across all subjects for both FE and PS. The predictions were generally better for FE than for PS. There were no statistically significant differences across the four categories of movements for $\operatorname{RMSE}_{\mathrm{FE}}(p=0.06), \operatorname{RMSE}_{\mathrm{PS}}(p=0.09)$, and $R_{\mathrm{PS}}^{2}(p=$ 0.35 ) while $R_{\mathrm{FE}}^{2}$ did show a significant difference ( $p=$ 0.01 ), with the performance during simulated ADLs being lower than during single-joint movements without a load and simultaneous/serial joint movements. Addi- tionally, there was no significant difference in predictive accuracy of the single-joint movements performed with a load in the hand relative to single-joint movements without load for any metric RMSE $_{\mathrm{FE}}[p>0.99]$, RMSE $\mathrm{PS}$ [p=0.63], $R_{\mathrm{FE}}^{2}[p>0.99]$, and $\left.R^{2} \mathrm{PS}[p>0.99]\right)$.

\section{Muscle Subset Selection}

To identify which muscles contain the most relevant information for joint-angle prediction, we evaluated EMG features from different combinations of muscles as TDANN inputs using a forward-selection algorithm. A TDANN with 20 hidden layer neurons and 7 input delays was trained to test each subset of inputs, repeated with 5 different sets of testing data. Features from all seven channels were tested individually and we first selected the single channel that produced the best prediction performance on the testing data set. We then evaluated prediction with the remaining six channels paired individually with the first channel, choosing the second most effective 
JRRD, Volume 48, Number 6, 2011

Table 1.

Summary of time-delayed artificial neural network prediction performance across all subjects $(N=5)$ and all movement types.

\begin{tabular}{|c|c|c|c|c|c|c|c|c|}
\hline \multirow{2}{*}{$\begin{array}{l}\text { Movement } \\
\text { Type }\end{array}$} & \multicolumn{2}{|c|}{$\begin{array}{c}\text { Single Joint } \\
\text { (without load) }\end{array}$} & \multicolumn{2}{|c|}{$\begin{array}{l}\text { Single Joint } \\
\text { (with load) }\end{array}$} & \multicolumn{2}{|c|}{ Simultaneous/Serial Joint } & \multicolumn{2}{|c|}{ Simulated ADLs } \\
\hline & RMSE ( $\left.{ }^{\circ}\right)$ & $R^{2}$ & RMSE ( $\left.{ }^{\circ}\right)$ & $R^{2}$ & RMSE $\left(^{\circ}\right)$ & $R^{2}$ & RMSE ( $\left.{ }^{\circ}\right)$ & $R^{2}$ \\
\hline \multicolumn{9}{|l|}{ Subject 1} \\
\hline $\mathrm{FE}$ & 12.8 & 0.67 & 16.2 & 0.77 & 13.8 & 0.89 & 11.1 & 0.74 \\
\hline PS & 25.0 & 0.53 & 25.4 & 0.54 & 24.0 & 0.62 & 19.8 & 0.64 \\
\hline \multicolumn{9}{|l|}{ Subject 2} \\
\hline \multicolumn{9}{|l|}{ Subject 3} \\
\hline $\mathrm{FE}$ & 13.0 & 0.88 & 15.3 & 0.88 & 16.0 & 0.83 & 19.8 & 0.71 \\
\hline PS & 29.2 & 0.40 & 34.5 & 0.37 & 34.5 & 0.22 & 29.7 & 0.42 \\
\hline \multicolumn{9}{|l|}{ Subject 4} \\
\hline FE & 12.7 & 0.87 & 17.9 & 0.73 & 18.6 & 0.83 & 20.8 & 0.56 \\
\hline PS & 14.0 & 0.74 & 19.8 & 0.56 & 22.1 & 0.53 & 23.2 & 0.54 \\
\hline \multicolumn{9}{|l|}{ Mean \pm SD } \\
\hline $\mathrm{FE}$ & $11.6 \pm 2.4$ & $0.83 \pm 0.10$ & $13.6 \pm 4.3$ & $0.81 \pm 0.06$ & $13.4 \pm 4.2$ & $0.86 \pm 0.03$ & $16.7 \pm 5.1$ & $0.70 \pm 0.08$ \\
\hline PS & $21.7 \pm 6.0$ & $0.51 \pm 0.15$ & $24.6 \pm 6.3$ & $0.50 \pm 0.09$ & $26.4 \pm 4.8$ & $0.39 \pm 0.22$ & $24.1 \pm 3.7$ & $0.47 \pm 0.11$ \\
\hline
\end{tabular}

muscle. Then the remaining five channels were paired with the best two channels, and so on. We repeated this procedure for both DOFs (FE and PS). Furthermore, this procedure was performed using two different performance metrics (RMSE and $R^{2}$ ) to guide the selection process, resulting in four distinct ordinal muscle sets for each subject (i.e., muscles selected to optimize FE or PS predictions and muscles selected to minimize RMSE or maximize $R^{2}$ ).

Figure 5 shows the ability of the TDANN to predict both elbow FE and forearm PS as a function of the number of muscles used as inputs, using both RMSE (upper panel) and $R^{2}$ (lower panel) as the performance metric. In general, Figure 5 shows a trend of increasing performance with an increasing number of muscles included in the set across both motions and both performance metrics $(p<0.001)$. There was no significant change in $R^{2}{ }_{\mathrm{PS}}(p>$ 0.99 ) when including more than four muscles, while performance plateaued at three muscles for $\operatorname{RMSE}_{\mathrm{FE}}(p>$ $0.99), \operatorname{RMSE}_{\mathrm{PS}}(p>0.23)$, and $R_{\mathrm{FE}}^{2}(p>0.99)$.

The relative contribution of the EMG signals from different muscles to the joint-angle predictions varied across subjects, i.e., the "rank" of each muscle in the forward-selection process was different across subjects.
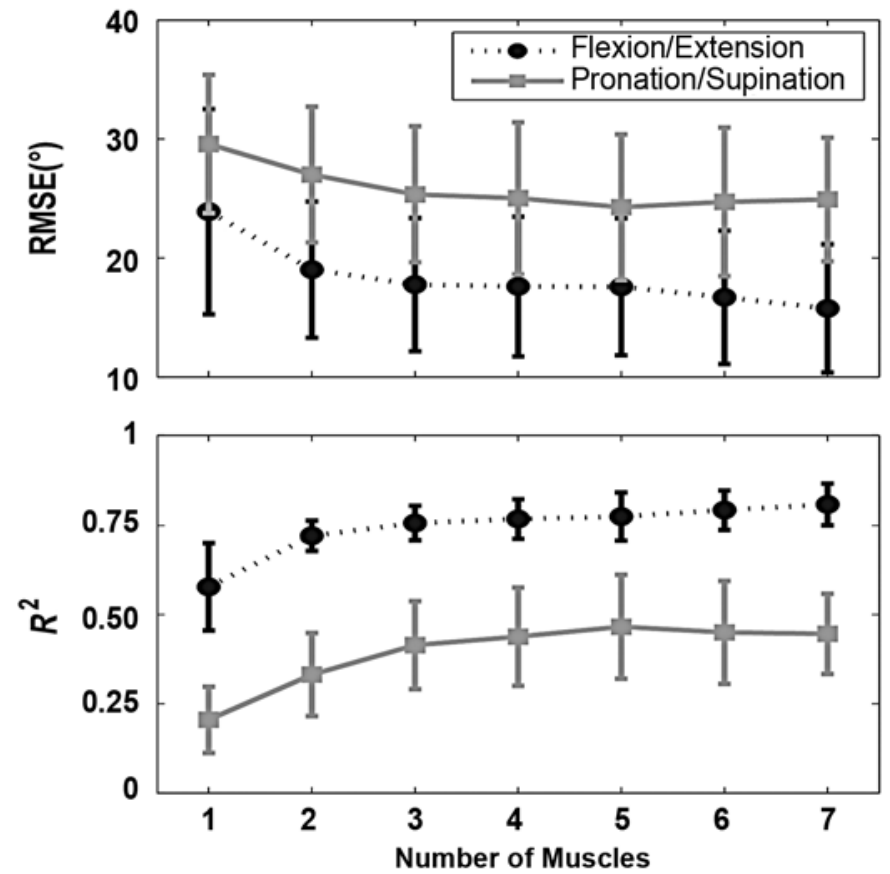

Figure 5.

Cross-validated time-delayed artificial neural network prediction performance as function of number of muscles that were provided as input to network. Muscles included were customized to each subject and selected using forward selection method. RMSE = root-mean-square error. 
To determine the average importance of each of the muscles, we averaged the rank of the muscles across all subjects. Table 2 illustrates the results, which show the average rank across subjects for each muscle when different performance metrics were used to guide the forwardselection algorithm that ranked the muscles. These performance metrics (each illustrated in a column in Table 2) were the RMSE of FE only, RMSE of PS only, average RMSE across both FE and PS, and corresponding three measures for $R^{2}$. In all cases, a single TDANN with 20 hidden layer neurons and 7 input delays was trained to predict both joint angles. The last column is an overall "meta"-average across all six metrics. It shows that biceps, brachialis, and triceps were generally selected early in the process (and as such have lower average ranks in Table 2), and that the three different heads of the deltoid and the clavicular pectoralis had very similar average ranks (indicating that they were not added with any particular order across all subjects).

As a practical matter, using a fixed set of muscle EMG signals in a clinical system rather than to customize a set for each patient would be desirable. Figure 6 illustrates the effect of using a fixed set of muscles based on average overall population predictive performance rather than using muscle sets specifically tailored to each subject using the forward selection method. The muscles selected in Figure 6 were the muscles that had the best average rank across all subjects using the average of the six different performance metrics (i.e., the values in the last column of Table 2). For example, biceps had the lowest average rank; therefore, they were used for evaluating the set of muscles with only one muscle for all subjects. Brachialis had the second lowest rank, so for evaluation of the subset with two muscles, brachialis and biceps were used, and so on. Figure 6 shows the TDANN prediction performance when features extracted from the fixed subsets were used as inputs compared with when features from the variable subject-specific subsets were used as inputs. For FE, there is no significant difference in performance ( $R^{2}$ or RMSE) between the fixed and subject-specific subsets once two or more muscles are included $(p>0.43)$. For PS, there is no significant difference in performance between the fixed and variable subsets for any number of muscles $(p>0.36)$.

\section{DISCUSSION}

Our long-term goal is to develop an implanted telemetry system and external controller for transhumeral prostheses that will use information extracted from EMG recordings to predict the prosthesis motor commands needed to make natural movements involving simultaneous control of multiple DOFs. Specifically, a TDANN structure was optimized and then successfully trained to simultaneously predict FE and PS movement trajectories recorded from nondisabled subjects using features extracted from windows of EMG as inputs. While they will need to be verified in a larger group of subjects, the results of this pilot study suggest that using EMG signals from muscles that would be available for recording in patients with transhumeral amputation to predict relevant arm motions (FE and PS) for a prosthetic limb is feasible.

\section{Neural Network Optimization and Prediction Performance}

In this proposed TDANN, time-domain features [4] were extracted from windows (128 ms wide with 50\% overlap) of EMG recordings and provided as inputs to the network. We did not systematically vary the window size or the amount of overlap, so it may be possible that

Table 2.

Mean \pm standard deviation ranks for each muscle using various performance metrics to guide forward selection process. Last column indicates overall average rank for each muscle across all six performance metrics.

\begin{tabular}{|c|c|c|c|c|c|c|c|}
\hline $\begin{array}{r}\text { Muscle } \\
\end{array}$ & RMSE $_{\text {FE }}$ & RMSE $_{\mathbf{P S}}$ & RMSE $_{\text {Avg }}$ & $R_{\text {FE }}^{2}$ & $R^{2}$ PS & $\boldsymbol{R}^{2}$ Avg & Overall \\
\hline Biceps & $2.8 \pm 1.3$ & $1.4 \pm 0.9$ & $1.6 \pm 0.5$ & $2.8 \pm 1.3$ & $1.2 \pm 0.4$ & $1.8 \pm 0.4$ & $1.9 \pm 0.7$ \\
\hline Brachialis & $2.4 \pm 1.9$ & $3.2 \pm 1.6$ & $2.6 \pm 2.1$ & $2.4 \pm 1.9$ & $3.2 \pm 1.6$ & $2.4 \pm 2.1$ & $2.7 \pm 0.4$ \\
\hline Triceps & $3.2 \pm 2.2$ & $3.4 \pm 2.1$ & $3.8 \pm 2.1$ & $3.4 \pm 2.3$ & $4.0 \pm 2.5$ & $3.8 \pm 2.6$ & $3.6 \pm 0.3$ \\
\hline Posterior Deltoid & $3.6 \pm 1.9$ & $6.0 \pm 1.0$ & $5.4 \pm 1.8$ & $3.6 \pm 1.9$ & $5.2 \pm 1.5$ & $5.2 \pm 1.5$ & $4.8 \pm 1.0$ \\
\hline Anterior Deltoid & $5.6 \pm 1.1$ & $4.4 \pm 1.5$ & $5.0 \pm 1.9$ & $6.2 \pm 0.8$ & $4.4 \pm 1.5$ & $5.2 \pm 1.5$ & $5.1 \pm 0.7$ \\
\hline
\end{tabular}



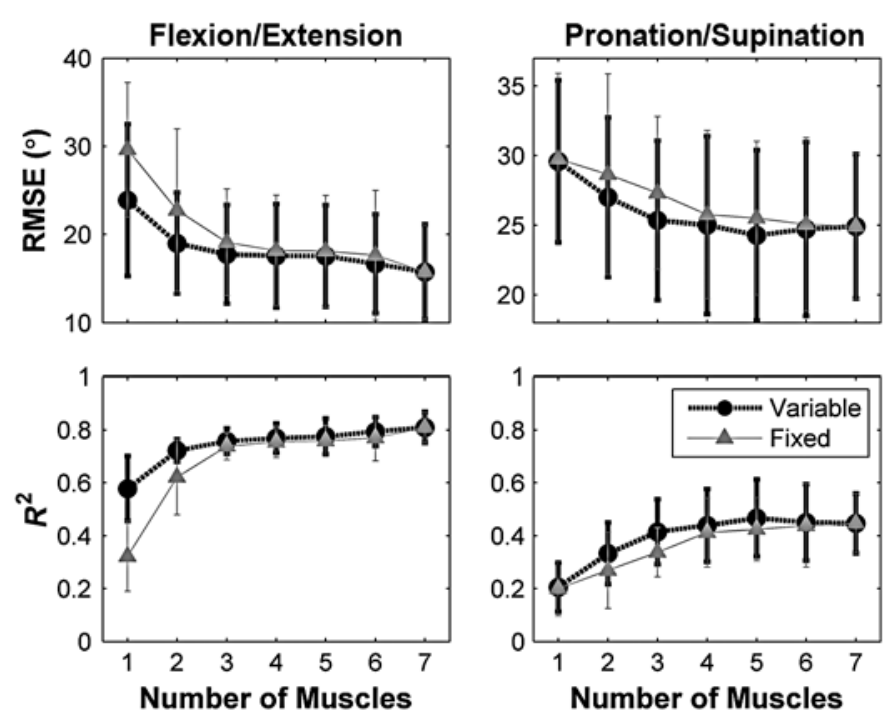

Figure 6.

Time-delayed artificial neural network prediction performance using different muscle subsets. Black circles show subsets tailored to each subject (variable). Gray triangles show subsets chosen based on results across all subjects (fixed). RMSE = root-mean-square error.

further optimization could improve prediction results. Evaluation of additional EMG features is another avenue for further refinement, as previous studies have shown that the inclusion of wavelet coefficients and autoregressive coefficients may provide modest gains in EMG pattern-classification accuracy [12].

Our results (Figure 3 ) indicate that a modestly sized TDANN with 10 to 20 hidden layer neurons was sufficient for joint-angle prediction using these time-domain features. Approximately $450 \mathrm{~ms}$ of input time history was shown to be useful in prediction. This finding is consistent with the 1 to $2 \mathrm{~Hz}$ mechanical natural frequency of the elbow [23]. Note that the inclusion of this input time history would not translate to a $450 \mathrm{~ms}$ pure delay perceived by the patient-it simply makes the current TDANN output a function of the current input as well as inputs occurring across the previous $450 \mathrm{~ms}$. Any perceived delay in our proposed scheme would be from EMG signal collection, windowing and feature extraction, and ANN computations. With overlapping windows for feature extraction, the total perceived delay should be within the suggested guidelines (i.e., 100-300 ms) for prosthesis controllers [3,24].

Since the selection of this "optimal" architecture was based on detecting statistically significant improvements in the predictions (i.e., increases in $R^{2}$ and decreases in RMSE) as a function of these parameters, identifying a different architecture with larger subject numbers might be possible. We might expect errors on the testing data to eventually increase if we continue to increase the number of neurons or delays caused by memorization of the training data rather than formation of a general model (i.e., overfitting). Prior to the errors increasing, there is a range of architectures that provide statistically equivalent performance. With the data collected in this study, we attempted to identify the smallest such network architecture to use in subsequent analyses. While we believe that our general findings related to the prediction of FE and PS are likely to be consistent, additional subjects will need to participate to verify that the number of hidden layer neurons and input time delays will generalize for the overall population.

Across a range of movements, TDANNs were able to predict the elbow FE and forearm PS joint angles from EMG recordings (Table 1). Average RMSEs across all subjects were approximately $15.7^{\circ}$ and $24.9^{\circ}$, with predicted trajectories having average $R^{2}$ values of approximately 0.81 and 0.46 for elbow FE and forearm PS, respectively. The RMSE for elbow FE is consistent with that demonstrated by Au and Kirsch [19], who also demonstrated the feasibility of using an EMG-based neural network approach to decoding more proximal movements such as humeral rotation, which is a frequently overlooked lost movement in the population with aboveelbow amputation. We would expect the predictions for FE to be superior to those for PS, because the EMG features from most of the primary muscles (i.e., biceps, brachialis, and triceps) involved in that movement were provided as inputs to the network. The only intact muscle directly involved in PS for a patient with transhumeral amputation is the biceps, which also acts as an elbow flexor; the other muscles involved in PS (i.e., supinator, pronator quadratus, and pronator teres) would all be missing following a transhumeral amputation. Given the initial results presented here, it is likely that the prediction of PS will need to be improved for the proposed scheme to be functional. It is possible that, with training, patients may be able to learn to modulate the activation of the residual musculature to control PS. Additionally, employing this scheme with patients who have undergone targeted muscle reinnervation [25] may improve PS prediction by providing information directly related to the activity of these missing muscles. 
The accuracy of PS predictions was consistent across all categories of movement. The errors for FE were larger during simulated ADLs than single-joint movements without a load and simultaneous/sequential joint movements. The detection of these differences in performance across classes of movement is affected, however, by limited statistical power because of the small number of subjects that participated in this pilot study.

Note that the results presented here used offline analysis of experimentally recorded data is important. That is, subjects were not provided with the opportunity to observe TDANN prediction errors during the experiments and to then alter their activation patterns to improve the predictions. We believe that having a user "in the loop" to correct for errors would result in better performance, but only further testing will evaluate this assumption. The relatively short duration of the experiment did not enable us to evaluate whether or not the performance of the proposed scheme would degrade or improve over time. An adaptive algorithm, however, could potentially be employed to address any variations in system performance. Our experiments also used nondisabled subjects with fully intact muscles. Impaired proprioceptive and tactile feedback may impair a person with amputation's ability to generate consistent patterns of muscle activation, which is crucial for the proposed control scheme to be functional. Other studies involving pattern recognition have shown that performance of these methods shows a significant decrease when applied to signals gathered from the amputated limb relative to those gathered from the nondisabled limb in patients with transradial amputation [26]. Future testing will be required to evaluate the ability of patients with amputation to control their multijoint myoelectric prosthesis using the proposed scheme.

\section{Input Muscle Selection}

While the EMG signals were recorded from a set of seven muscles in the arm and shoulder during experimental sessions, not all muscles made a significant contribution to the ability of the neural network to predict the movements. To identify which muscles contained the most relevant information, we evaluated muscle subsets using a forward-selection algorithm. Figure 5 shows a trend of improving performance with an increased number of muscles included as inputs to the TDANN, but this improvement in performance saturated after three or four muscles. We recognize that the order in which muscles were identified may vary with the choice of a different selection algorithm (such as a backward elimination approach) but believe that our general findings are likely to be consistent regardless of algorithm choice.

While the results of the forward-selection process would suggest the need for subject-specific muscle sets for optimal control, identifying a standard set of muscles that would be generally useful across different patients with an above-elbow amputation may be desirable. We found no significant difference between a fixed muscle set (chosen based on population statistics as shown in Table 2) and the subject-tailored muscle sets when looking at sets that contained two (biceps and brachialis) or more muscles. Additionally, once two more muscles (triceps and one head of the deltoid) were added to the fixed set, there was no significant difference between the performance of this four-muscle subset and the performance using all seven muscles (Figure 6), suggesting that the remaining muscles did not add significant new information for prediction of the FE and PS joint angles.

One potentially confounding factor was that the brachialis was the only muscle for which we used a fine-wire intramuscular electrode. Our original intent was to sample all channels intramuscularly. Subjects participating in an earlier study, however, reported significant levels of discomfort when intramuscular electrodes were inserted into the biceps, triceps, and brachialis. As their range of motion was limited by the presence of just three fine-wire electrodes, we did not proceed with recording from the remainder of the muscles in this fashion. The EMG signals from the rest of the muscles considered in this study (biceps; triceps; anterior, middle, and posterior deltoid; and clavicular pectoralis major) are reliably accessible in a laboratory setting with surface electrodes, and manual muscle tests were used to verify placement at the onset of each experiment. While it is possible that the relative contribution of the brachialis would have been different if we had used intramuscular electrodes for all seven muscles, we do not believe that this would change our general findings regarding the input muscle set. The fundamental goals of this study were to (1) examine the feasibility of decoding the movement trajectories using the natural patterns of activation in the remaining musculature and (2) identify muscles containing key information. Given the subject discomfort and its effect on range of motion, we believed that we could achieve these goals by using a combination of intramuscular (when necessary) and surface electrodes in this laboratory study. However, many of 
the muscles considered would not be accessible in a clinical system using currently available surface technologies - whether they are deeper muscles (e.g., brachialis) or muscles that would not be located directly beneath the socket (e.g., deltoid). A chronically implanted EMG recording system will be necessary to realize the proposed control approaches. Noting that the findings may not be directly relevant for patients with short transhumeral amputation is also important; the brachialis may not be intact because its origin is more distal than the triceps or biceps.

\section{Options for Controlling Terminal Device}

In addition to a prosthetic elbow and wrist rotator, a command interface for transhumeral prostheses must also include operating a terminal device (i.e., hand) for grasping. Our study, however, did not directly address this important issue. Achieving robust and natural MES control of the hand is not likely for this patient population because of the loss of all relevant hand musculature. Users of body-powered transhumeral prostheses frequently use movements of the shoulder to operate their terminal devices with a harness and cable system. We believe that an analogous myoelectric hand controller could be devised whereby a patient would modulate the EMG signals from shoulder girdle muscles (e.g., trapezius) to open and close the hand. Combining such a scheme with the neural network controller developed in this study could enable patients to operate all three joints of their prostheses in a simultaneous and coordinated fashion, but future work is necessary to assess the feasibility of this approach.

\section{CONCLUSIONS}

This feasibility study demonstrated that an appropriately designed TDANN is capable of predicting continuous and simultaneous elbow FE and forearm PS joint angles, with average RMSEs of approximately $15.7^{\circ}$ and $24.9^{\circ}$ and average $R^{2}$ values of approximately 0.81 and 0.46 , respectively, in nondisabled subjects using features extracted from EMG signals obtained from muscles that would remain following a transhumeral amputation. These results indicate that the signals contain a significant amount of information related to arm movements. With further development, it may be possible to not only control these two DOFs simultaneously, but to do so with enhanced accuracy, which would enable patients to make more natural, coordinated movements and improve their ability to perform ADLs.

\section{ACKNOWLEDGMENTS}

\section{Author Contributions:}

Study concept and design: C. L. Pulliam, J. M. Lambrecht, R. F. Kirsch. Acquisition, analysis, and interpretation of data: C. L. Pulliam. Drafting of manuscript: C. L. Pulliam. Critical revision of manuscript for important intellectual content: C. L. Pulliam, J. M. Lambrecht, R. F. Kirsch. Obtained funding: R. F. Kirsch.

Administrative, technical, or material support: J. M. Lambrecht, R. F. Kirsch.

Study supervision: R. F. Kirsch.

Financial Disclosures: The authors have declared that no competing interests exist.

Funding/Support: This material was based on work supported by the Department of Defense Telemedicine and Advanced Technology Research Center (grant W81XWH-07-2-0044) and the National Institutes of Health National Institute of Biomedical Imaging and Bioengineering (grant T32-EB04314).

Additional Contributions: The authors would like to acknowledge the assistance of Dr. S. Huang for performing the intramuscular insertions, P. Cooman and Dr. A. B. Ajiboye for reviewing the manuscript, and B. Memberg for his efforts on regulatory issues.

Institutional Review: All subjects gave informed consent to the procedures as approved by the MetroHealth Medical Center Institutional Review Board.

Participant Follow-Up: The authors plan to inform participants of the publication of this study.

\section{REFERENCES}

1. Adee S. The revolution will be prosthetized-DARPA's prosthetic arm gives amputees new hope. IEEE Spectrum. 2009 January:44-48. DOI:10.1109/MSPEC.2009.4734314

2. Biddiss EA, Chau TT. Upper limb prosthesis use and abandonment: A survey of the last 25 years. Prosthet Orthot Int. 2007;31(3):236-57. [PMID: 17979010] DOI:10.1080/03093640600994581

3. Englehart K, Hudgins B. A robust, real-time control scheme for multifunction myoelectric control. IEEE Trans Biomed Eng. 2003;50(7):848-54. [PMID: 12848352] DOI:10.1109/TBME.2003.813539

4. Hudgins B, Parker P, Scott RN. A new strategy for multifunction myoelectric control. IEEE Trans Biomed Eng. 1993; 40(1):82-94. [PMID: 8468080]

DOI:10.1109/10.204774

5. Graupe D, Magnussen J, Beex AA. A microprocessor system for multifunctional control of upper-limb prostheses via 
myoelectric signal identification. IEEE Trans Auto Control. 1978;23(4):538-44. DOI:10.1109/TAC.1978.1101783

6. Englehart K, Hudgins B, Parker PA. A wavelet-based continuous classification scheme for multifunction myoelectric control. IEEE Trans Biomed Eng. 2001;48(3):302-11.

[PMID: 11327498]

DOI:10.1109/10.914793

7. Lawrence PD, Kadefors R. Classification of myoelectric patterns for the control of a prosthesis. In: Herberts $\mathrm{P}$, Kadefors R, Magnusson R, Petersen I, editors. The control of upper-extremity prostheses and orthoses. Springfield (IL): Thomas; 1974.

8. Kilgore KL, Hoyen HA, Bryden AM, Hart RL, Keith MW, Peckham PH. An implanted upper-extremity neuroprosthesis using myoelectric control. J Hand Surg Am. 2008;33(4): 539-50. [PMID: 18406958]

DOI:10.1016/j.jhsa.2008.01.007

9. Dutta A, Kobetic R, Triolo RJ. Gait initiation with electromyographically triggered electrical stimulation in people with partial paralysis. J Biomech Eng. 2009;131(8): 081002. [PMID: 19604014$]$

DOI:10.1115/1.3086356

10. Weir RF, Troyk PR, DeMichele GA, Kerns DA, Schorsch JF, Maas H. Implantable myoelectric sensors (IMESs) for intramuscular electromyogram recording. IEEE Trans Biomed Eng. 2009;56(1):159-71. [PMID: 19224729] DOI:10.1109/TBME.2008.2005942

11. Farrell TR, Weir RF. A comparison of the effects of electrode implantation and targeting on pattern classification accuracy for prosthesis control. IEEE Trans Biomed Eng. 2008;55(9):2198-2211. [PMID: 18713689]

DOI:10.1109/TBME.2008.923917

12. Hargrove LJ, Englehart K, Hudgins B. A comparison of surface and intramuscular myoelectric signal classification. IEEE Trans Biomed Eng. 2007;54(5):847-53.

[PMID: 17518281]

DOI:10.1109/TBME.2006.889192

13. Saridis GN, Gootee TP. EMG pattern analysis and classification for a prosthetic arm. IEEE Trans Biomed Eng. 1982; 29(6):403-12. [PMID: 7106790]

DOI:10.1109/TBME.1982.324954

14. Ajiboye AB, Weir RF. A heuristic fuzzy logic approach to EMG pattern recognition for multifunctional prosthesis control. IEEE Trans Neural Syst Rehabil Eng. 2005;13(3): 280-91. [PMID: 16200752] DOI:10.1109/TNSRE.2005.847357

15. Chan FH, Yang YS, Lam F, Zhang YT, Parker PA. Fuzzy EMG classification for prosthesis control. IEEE Trans Rehabil Eng. 2000;8(3):305-11. [PMID: 11001510] DOI:10.1109/86.867872

16. Kelly M, Parker PA, Scott RN. Myoelectric signal analysis using neural networks. IEEE Eng Med Biol Mag. 1990:61-64.
[PMID: 18238322]

DOI:10.1109/51.62909

17. Cheron G, Draye JP, Brougeios M, Libert G. A dynamic neural network identification of electromyography and arm trajectory relationship during complex movements. IEEE Trans Biomed Eng. 1995;43(5):552-58. [PMID: 8849468] DOI:10.1109/10.488803

18. Sebelius F, Eriksson L, Balkenius C, Laurell T. Myoelectric control of a computer animated hand: A new concept based on the combined use of a tree-structured artificial neural network and a data glove. J Med Eng Technol. 2006; 30(1):2-10. [PMID: 16393847] DOI:10.1080/03091900512331332546

19. Au AT, Kirsch RF. EMG-based prediction of shoulder and elbow kinematics in able-bodied and spinal cord injured individuals. IEEE Trans Rehabil Eng. 2000;8(4):471-80. [PMID: 11204038]

DOI:10.1109/86.895950

20. Hincapie JG, Blana D, Chadwick EK, Kirsch RF. Musculoskeletal model-guided, customizable selection of shoulder and elbow muscles for a C5 SCI neuroprosthesis. IEEE Trans Neural Syst Rehabil Eng. 2008;16(3):255-63.

[PMID: 18586604] DOI:10.1109/TNSRE.2008.922681

21. Cram JR, Kasman GS. Introduction to surface electromyography. Gaithersburg (MD): Aspen Publishers; 1998.

22. Bishop CM. Pattern recognition and machine learning. New York (NY): Springer; 2006.

23. Bennet DJ, Hollerbach JM, Xu Y, Hunter IW. Time-varying stiffness of human elbow joint during cyclic voluntary movement. Exp Brain Res. 1992;88(2):433-42.

[PMID: 1577114$]$

DOI:10.1007/BF02259118

24. Farrell TR, Weir RF. The optimal controller delay for myoelectric prostheses. IEEE Trans Neural Syst Rehabil Eng. 2007;15(1):111-18. [PMID: 17436883]

DOI:10.1109/TNSRE.2007.891391

25. Kuiken TA, Dumanian GA, Lipschutz RD, Miller LA, Stubblefield KA. The use of targeted muscle reinnervation for improved myoelectric prosthesis control in a bilateral shoulder disarticulation amputee. Prosthet Orthot Int. 2004; 28(3):245-53. [PMID: 15658637]

26. Li G, Schultz AE, Kuiken TA. Quantifying pattern recognition-based myoelectric control of multifunctional transradial prostheses. IEEE Trans Neural Syst Rehabil Eng. 2010;18(2):185-92. [PMID: 20071269]

DOI:10.1109/TNSRE.2009.2039619

Submitted for publication December 17, 2010. Accepted in revised form May 9, 2011. 
JRRD, Volume 48, Number 6, 2011

This article and any supplementary material should be cited as follows:

Pulliam CL, Lambrecht JM, Kirsch RF. Electromyogrambased neural network control of transhumeral prostheses.
J Rehabil Res Dev. 2011;48(6):739-54.

DOI:10.1682/JRRD.2010.12.0237 